\title{
Fault Diagnosis System Identification Based on Impedance Matching Balance Transformer
}

\author{
Yanjun Ren ${ }^{*}$ and Xinli Deng \\ Chongqing Radio \& TV University, Chongqing 400052, China \\ *Corresponding author
}

\begin{abstract}
Impedance matching balance transformer is often used as the main electrified railway traction substation transformer. In order to solve the problem that traditional fault diagnosis method could not accurately identify the internal or external failure, it is proposed an identification algorithm of recursive least square parameter estimation online to distinguish the internal fault from external fault, which is based on that the winding resistance and short circuit reactance values have not been changed in normal operation for impedance matching balance transformer or external fault, but they have been changed for internal fault. The curves of simulation proved that the proposed algorithm could effectively distinguish internal fault from external fault. The research results show the feasibility to identify two sorts of different faults.
\end{abstract}

Keywords-system identification; impedance matching balance transformer; fault diagnosis

\section{INTRODUCTION}

Impedance matching balance transformer with transformation and commutation is the main electrified railway traction substation transformer[1]. Transformer's stable operation is directly related to the safety of high-speed rail, so it is very important to accurately distinguish possible fault types and design different protection devices[2]. Transformers of electrified railway power supply system work under poor conditions. Currently, microcomputer protection devices in operation are almost designed based on the principle of differential protection. In general, it is difficult to distinguish to be internal failure or external fault, which may lead to protection tripping or malfunction and then endanger the safe operation of electrified railway system[3]. Based on that the winding resistance and short circuit reactance values could not been changed in normal operation for impedance matching balance transformer or external fault while the winding resistance and short circuit reactance values would changed dramatically, the winding resistance and short circuit reactance values of transformer could be online identified[4]. By comparing the identification values with the normal parameter values, we can determine whether the impedance matching balance transformer protection action is required[5].

\section{IDENTIFICATION MATHEMATICAL MODEL OF IMPEDANCE MATCHING BALANCE TRANSFORMER}

Figure I shows the winding resistance of impedance matching balance transformer[6]. According to the operational principle of impedance matching balance transformer, we know that its high voltage side is connected into a star connection, the low voltage side is connected into triangle junction and the iron core limb winding in B phase of low voltage side extend 2 supporting arms, called balance winding. From the figure 1, we can see that the core limb in A phase and C phase of impedance matching transformer winds two windings and that in $\mathrm{B}$ phase winds four windings. In accordance to the principle of the multi-winding transformer circuit, magnetic circuit and equivalent circuit, the linear model of impedance matching balance transformer in equation (1) can be obtained through a series of simplified processing [7]. In equation (1), $\mathrm{K}=\mathrm{N} 1 / \mathrm{N} 2$ and $\mathrm{x} 1$ is the short circuit reactance of high voltage side, other notations see the reference [8].

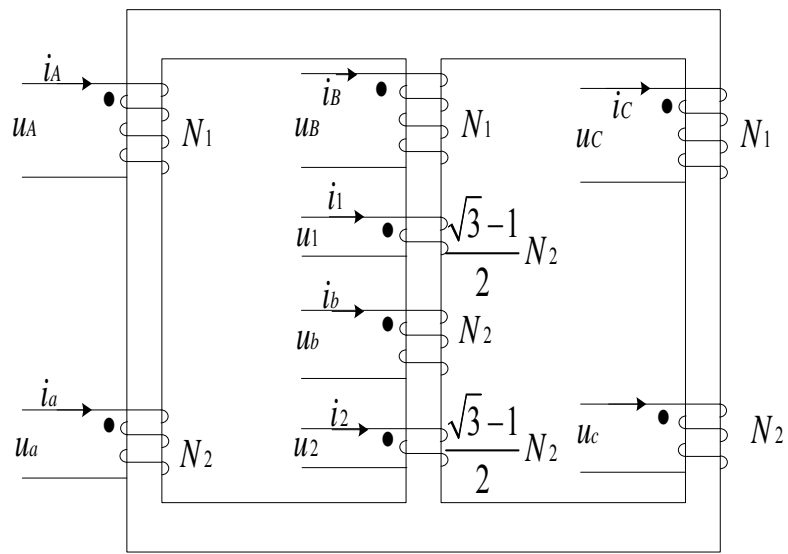

FIGURE I. THE WINDING OF IMPEDANCE MATCHING BALANCE TRANSFORMER

From the equation (1), we can see that $u_{A}, u_{B}$ and $u_{C}$ are the voltage values of three phases at the high voltage side. $i_{A}, i_{B}$ and $i_{C}$ are the current values of windings at the high voltage side. $u_{\alpha}, u_{\beta}$ and $i_{\alpha}, i_{\beta}$ are the voltage values and current values of two phases at the low voltage side respectively, which are directly treasured as the input and output of the transformer's parameter identification model. The winding resistance $\mathrm{R}$ and short circuit reactance $x_{1}$ values at the high voltage side as the parameters to be identified can constitute the grey box model of system identification, which can respectively construct A, B and $\mathrm{C}$ phases identification mathematical models of impedance matching balance transformer through discretization. This paper only with A-phase winding for example analyses the relevant fault diagnosis. 


$$
\left\{\begin{array}{l}
u_{A}-\frac{\sqrt{3}-1}{2} u_{B}-K u_{\alpha}=R i_{A}-\frac{\sqrt{3}-1}{2} R i_{B}+ \\
\frac{R}{K}\left(\frac{\sqrt{3}+1}{2 \sqrt{3}} i_{\alpha}-\frac{\sqrt{3}-1}{2 \sqrt{3}} i_{\beta}\right)+\frac{x_{1}}{2} \frac{d i_{A}}{d \varpi t}- \\
\frac{(5-2 \sqrt{3}) x_{1}}{8 \sqrt{3}} \frac{d i_{B}}{d \varpi t}+\frac{(16-3 \sqrt{3}) x_{1}}{24 K} \frac{d i_{\alpha}}{d \varpi t}+ \\
\frac{(7-4 \sqrt{3}) x_{1}}{24 K} \frac{d i_{\beta}}{d \varpi t} \\
u_{B}-\frac{K}{\sqrt{3}}\left(u_{\alpha}+u_{\beta}\right)=R i_{B}-\frac{R}{3 K}\left(i_{\alpha}+i_{\beta}\right)+ \\
\frac{2 x_{1}}{3} \frac{d i_{B}}{d \varpi t}-\left[\frac{5-7 \sqrt{3}}{12 K}+\frac{2-\sqrt{3}}{12 \sqrt{3} K{ }^{2}}\right] x_{1} \frac{d\left(i_{\alpha}+i_{\beta}\right)}{d \varpi t} \\
u_{C}-\frac{\sqrt{3}-1}{2} u_{B}-K u_{\beta}=R i_{C}-\frac{\sqrt{3}-1}{2} R i_{B}+ \\
\frac{R}{K}\left(\frac{\sqrt{3}+1}{2 \sqrt{3}} i_{\beta}-\frac{\sqrt{3}-1}{2 \sqrt{3}} i_{\alpha}\right)+\frac{x_{1}}{2} \frac{d i_{C}}{d \varpi t}- \\
\frac{(5-2 \sqrt{3}) x_{1}}{8 \sqrt{3}} \frac{d i_{B}}{d \varpi t}+\frac{(16-3 \sqrt{3}) x_{1}}{24 K} \frac{d i_{\beta}}{d \varpi t}+ \\
\frac{(7-4 \sqrt{3}) x_{1}}{24 K} \frac{d i_{\alpha}}{d \varpi t}
\end{array}\right.
$$

For online real-time detection the winding resistance and short reactance values of transformer, winding parameter identification model of transformer A-phase is as follows.

$$
\begin{gathered}
Z_{A}(n)=\frac{u_{A}[(n+1) \Delta T]+u_{A}[n \Delta T]}{2}-\frac{(\sqrt{3}-1)}{2} \frac{u_{B}[(n+1) \Delta T]+u_{B}[n \Delta T]}{2}- \\
K \frac{u_{\alpha}[(n+1) \Delta T]+u_{\alpha}[n \Delta T]}{2}
\end{gathered}
$$$$
h^{T}{ }_{A}(n)=\left[\frac{i_{A}[(n+1) \Delta T]+i_{A}[n \Delta T]}{2}-\frac{(\sqrt{3}-1)}{2} \frac{i_{B}[(n+1) \Delta T]+i_{B}[n \Delta T]}{2}\right.
$$$$
+\frac{\sqrt{3}+1}{2 \sqrt{3} K} \frac{i_{\alpha}[(n+1) \Delta T]+i_{\alpha}[n \Delta T]}{2}-\frac{\sqrt{3}-1}{2 \sqrt{3} K} \frac{i_{\beta}[(n+1) \Delta T]+i_{\beta}[n \Delta T]}{2},
$$$$
\frac{1}{2 \sigma} \frac{i_{A}[(n+1) \Delta T]-i_{A}[n \Delta T]}{\Delta T}-\frac{5-2 \sqrt{3}}{8 \sqrt{3} \sigma} \frac{i_{B}[(n+1) \Delta T]-i_{B}[n \Delta T]}{\Delta T}
$$$$
\left.+\frac{16-3 \sqrt{3}}{24 K \varpi} \frac{i_{\alpha}[(n+1) \Delta T]-i_{\alpha}[n \Delta T]}{\Delta T}+\frac{7-4 \sqrt{3}}{24 K \varpi} \frac{i_{\beta}[(n+1) \Delta T]-i_{\beta}[n \Delta T]}{\Delta T}\right]
$$

$$
\theta_{A}^{T}=\left[R, x_{1}\right]
$$

\section{FAUIT Diagnosis MEthod OF IMPEDANCE MATCHING BALANCE TRANSFORMER BASED ON RLS}

\section{A. Forgetting Factor Algorithm of Recursive least Square Parameter Estimation}

The characteristics of the least square technique is directly to use all the observed data have been obtained to compute and process[9]. Therefore, the parameters of system model need to been re-estimated if having the new observation data every time, which means that all the obtained observed data to estimate the parameters of the model needs to be stored. And the complexity of solving the matrix equation $\hat{\theta}=\left(H_{L}{ }^{T} \Lambda_{L} H_{L}\right)^{-1} H_{L}{ }^{T} \Lambda_{L} Z_{L}$ results in the difficulties of online identification. The solution of this problem is to be turned into recursive least square algorithm. The basic idea is as follows.

$$
\hat{\theta}(k)=\hat{\theta}(k-1)+\text { correction term }
$$

The characteristic of the method is to revise the last time estimated results with new obtained observational data and then recursively estimate the next time parameter results[10]. However, there will be "data saturation" phenomenon as the increase of data when the method applies to online real-time identification. In other words, as the growth of $k$, gain matrix $K$

(k) will gradually tends to zero so that it could not revise $\hat{\theta}(k)$. Forgetting factor method can solve "data saturation" phenomenon effectively. The basic idea is that the old data adds forgetting factor to reduce the amount of old data information and increase the amount of new data information. Forgetting factor recursive least square algorithm is as equation (4).

$$
\left\{\begin{array}{l}
\hat{\theta}(k)=\hat{\theta}(k-1)+K(k)\left[z(k)-h^{T}(k) \hat{\theta}(k-1)\right] \\
K(k)=P(k-1) h(k) /\left(h^{T}(k) P(k-1) h(k)+\mu\right) \\
P(k)=\frac{1}{\mu}\left[I-K(k) h^{T}(k)\right] P(k-1)
\end{array}\right.
$$

Where $\mu$ is forgetting factor, $0<\mu \leq 1, \mu$ is not usually less than 0.9 . $0.95 \leq \mu \leq 1$ will be selected if the process is linear[11]. The large forgetting factor will reduce the tracking performance of the algorithm, and the system will be easily affected by noise with the small forgetting factor.

\section{B. The Process of Parameter Identification and Initial Value Selection}

Taking A-phase as an example, the process of parameter identification can be analyzed by means of identification model (4). According to the measured data, $Z_{A}(n), h^{T}{ }_{A}(n)$, $\theta^{T}$ A can be obtained. Substituting recursive least square algorithm equation, we can identify the parameters. Figure II shows the program flow diagram of the algorithm. In figure II $\theta_{0}$ is the initial value of identification parameter, $\theta_{i k}$ is the identification parameter value at $k$ is the threshold. The simulation program of algorithm can be written using MATLAB[12]. 


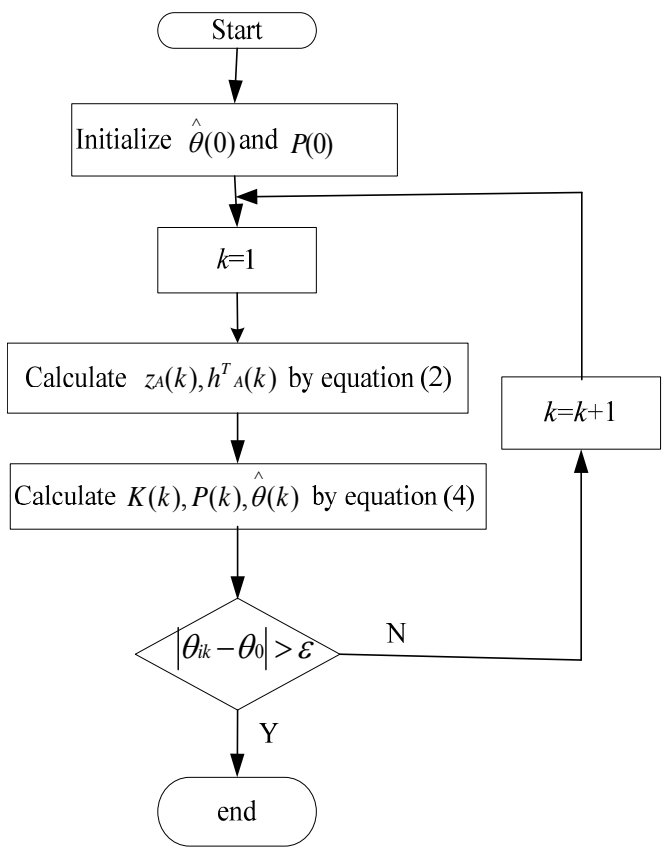

FIGURE II. THE PROGRAM FLOW DIAGRAM OF THE RLS ALGORITHM

In order to improve the protection sensitivity and rapidity of impedance matching balance transformer, the initial value of the algorithm is $P(0)=\alpha^{2} I$, where $\alpha$ is sufficiently large real number, $\hat{\theta}(0)$ is the parameter values of impedance matching balance transformer given by manufacturers, the forgetting factor $\mu$ is 0.95 .

\section{The Simulation AND ANALYsis}

The simulation employs a impedance matching balance transformer, whose capacity is 25MVA, rated voltage is $U_{1 N} / U_{2 N}=(100 \sqrt{3}) / 27.5 \mathrm{KV}$ and the short circuit reactance is $2.72+\mathrm{j} 25.06 \Omega \quad$, namely, $r_{A}=1.36 \Omega, x_{1}=25.06 \Omega$. During normal operation, the error of original and secondary side voltage transformer is $2 \%$, the error of original and secondary side current transformer is $10 \%$ and the errors of all the resistance and reactance parameters are $0.5 \%$. The original parameters $\theta^{T}=\left[r_{A}, X_{1}\right]$ given by the model of impedance matching balance transformer are used as the initial value of parameter values to be identified in simulation. Using electromagnetic transient simulation program (EMTP) we can get the real-time voltage and current sampling values of the impedance matching balance transformer windings on both sides. And then these values are used as the input and output of the identification model. The parameter values to be identified at every sampling time can be calculated by writing the program of recursive least square algorithm in MATLAB simulation software. Next the changes of impedance matching balance transformer parameter values to be identified will be analyzed in various operating conditions.

\section{A. Normal Operation}

Figure III and figure IV shows the identification curve graph of the winding resistance value $R$ and short circuit reactance value $x_{1}$ at the high voltage side when the impedance matching balance transformer is in normal operation.

In accordance to the parameters identification curve, we can see that when the impedance matching balance transformer is in normal operation, the resistance identification value of the winding at the high voltage side has a certain oscillation at $0.002 \mathrm{~s}$ ago but then quickly converge near $1.374 \mathrm{~s}$. At the same time, the short circuit reactance identification value at the high voltage side has a great change at first and then tends to be stable. We also can see that the winding resistance value $R$ and short circuit reactance value $x_{1}$ have been changed little in normal operation for impedance matching balance transformer at the high voltage side.

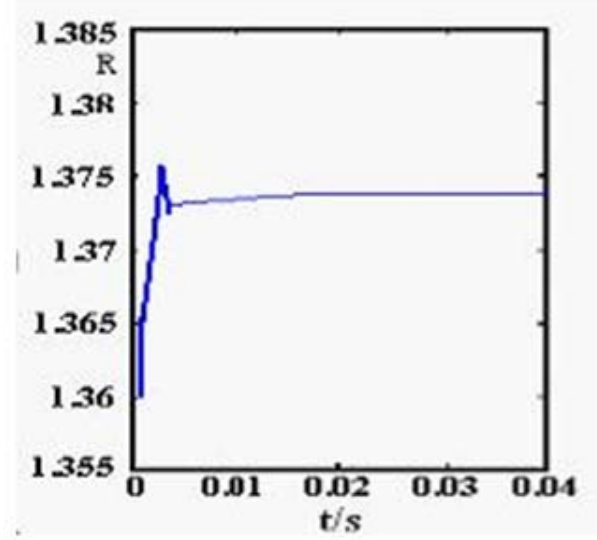

FIGURE III. THE IDENTIFICATION CURVE FOR RESISTANCE

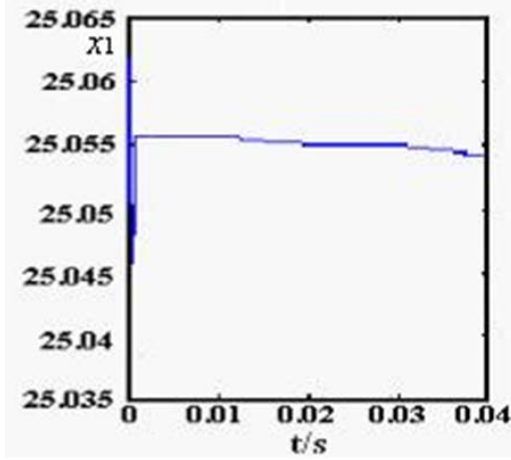

FIGURE IV. THE IDENTIFICATION CURVE FOR SHORT CIRCUIT REACTANCE

\section{B. External $\alpha$ Phase Winding Shorts to Ground}

Earth short circuit of external $\alpha$ phase winding at the secondary side (external fault) suddenly occurred at $0.002 \mathrm{~s}$, when the impedance matching balance transformer is in normal operation. Under the circumstance, the identification curve graph of the winding resistance value $R$ and short circuit 
reactance value $x_{1}$ of impedance matching balance transformer at the high voltage side is shown as figure V and figure VI.

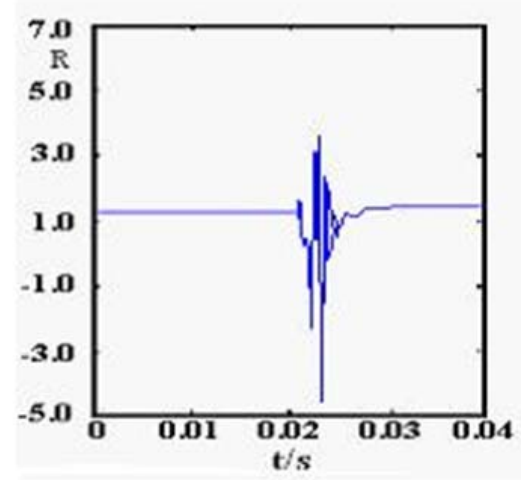

FIGURE V. THE IDENTIFICATION CURVE FOR RESISTANCE IN EXTERNAL fault

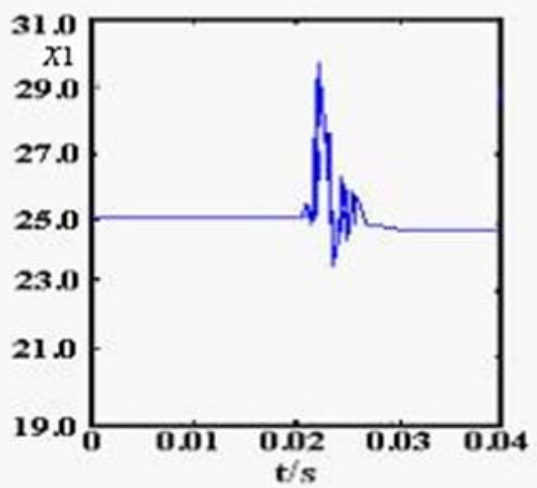

FIGURE VI. THE IDENTIFICATION CURVE FOR SHORT CIRCUIT REACTANCE IN EXTERNAL FAULT

As shown in figure 5 and figure 6 , the winding resistance value $R$ and short circuit reactance value $x_{1}$ at the high voltage side have oscillation when External $\alpha$ phase winding shorts to ground at $0.002 \mathrm{~s}$, but then the values tend to be stable and almost equal to them in normal operation.

\section{A Phase Winding at the High Voltage Side Shorts to Ground}

Single phase earth fault of A-phase winding at the high voltage side (internal fault) suddenly occurred at $0.002 \mathrm{~s}$ when the impedance matching balance transformer is in normal operation. Under the circumstance, the identification curve graph of the winding resistance value $R$ and short circuit reactance value $x_{1}$ of impedance matching balance transformer at the high voltage side is shown as figure VII and figure VIII.

As shown in figure VII and figure VIII, the winding resistance and short circuit reactance identification values at the high voltage side remain unchanged in normal operation for impedance matching balance transformer. The values change dramatically and will never converge when Single phase earth fault of A-phase winding at the high voltage side occurred at $0.002 \mathrm{~s}$.

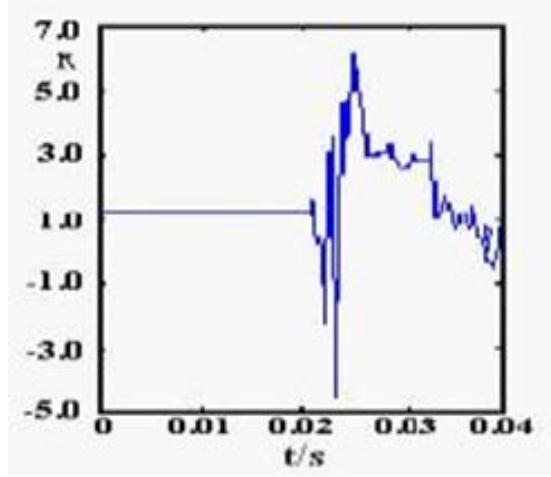

FIGURE VII. THE IDENTIFICATION CURVE FOR RESISTANCE IN INTERNAL fault

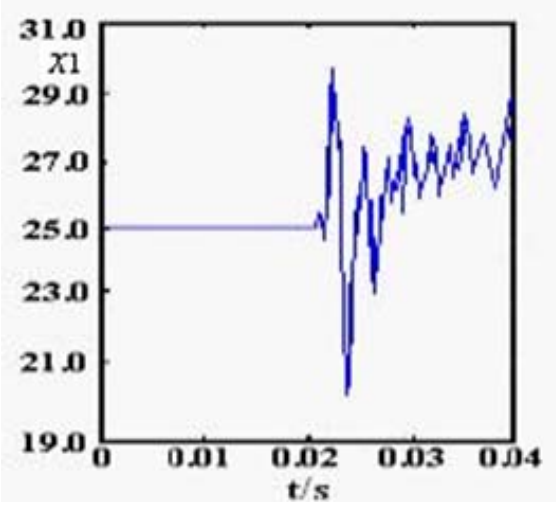

FIGURE VIII. THE IDENTIFICATION CURVE FOR SHORT CIRCUIT REACTANCE IN INTERNAL FAULT

The paper will not explain the inter-phase short circuit fault and inter-run short circuit fault of phase winding for impedance matching balance transformer, however, the results is similar to the above mentioned.

The simulation results show that the winding resistance and short circuit reactance values have not been significantly changed in normal operation for impedance matching balance transformer or external fault, but they have been dramatically changed for internal fault.

\section{CONCLUSION}

In order to solve the puzzle that the existing fault diagnosis methods could not accurately identify the types of fault for impedance matching balance transformer, the paper proposed a fault diagnosis method exploiting recursive least square algorithm to identify the internal failure and external fault based on operational feature of impedance matching balance transformer. The simulation results in MATLAB indicate that the proposed method could effectively make an accurate diagnosis of the internal and external fault.

\section{REFERENCES}

[1] IEEEGuide for Protective Relay Applications to Power Transformers, 2000 
[2] K. L. Butler-Purry and A. Kuforiji "Experimental results from short-circuit faults ona distribution transformer", Proc. IEEETransm. Distrib. Conf. Expo., vol. 1, pp.299 -306 2001 .

[3] K. L. Butler-Purry , M. Bagriyanik, M.J. Mousavi and P. Palmer-Buckle "Experimental investigation of internalshort circuit faults leading to advanced incipient behavior and failureof a distribution transformer", Proc.IEEE Power Syst. Conf. Expo., vol. 3, pp.1407 -1416 2004 .

[4] V. Behjat and A. Vahedi "An experimental approachfor investigating low-level interturn winding faults in power transformers", Elect. Eng., vol. 95, no. 2, pp. $135-1452013$.

[5] R. S. Bhide, M.S. S. Srinivas , A. Banerjee and R. Somakumar "Analysis of windinginter-turn fault in transformer: A review and transformer models", Proc. IEEE Int. Conf. Sustain. Energy Technol., pp.1 -7 2010.

[6] N. C. Joshi , Y.R. Sood , R.K. Jarial and R. Thapliyal "Transformer internal winding faultsdiagnosis methods: A review", MIT Int.J. Elect. Instrum. Eng., vol. 2, no. 2, pp.77 -81 2012.

[7] A. Bhoomaiah , P. K. Reddy , K. S. L. Murthy , P. A. Naidu and B. Singh "Measurement of neutralcurrents in a power transformer and fault detection using wavelettechniques", Proc. Annu. Rep. Conf.Elect. Insul. Dielectr. Phenomena, pp.170-1732004.

[8] M. Faridi , E. Rahimpour , M. Kharezi , H. R. Mirzaei and A. Akbari "Localization of turn-to-turnfault in transformers using artificial neural networks and windingtransfer function", Proc. IEEE 10thInt. Conf. Solid Dielectr., pp.1 -4 2010.

[9] V. Behjat and A. Vahedi "Numerical modeling of transformers interturn faultsand characterizing the faulty transformer behavior under various faultsand operating conditions", IET Elect.Power Appl., vol. 5, no. 5, pp.415 -431 2011.

[10] M. Vikash , K. Sharlinprija and M.K. Ilampoornan "Transformer fault detection by frequencyresponse analysis", Int. J. Comput.Sci. Elect. Eng., vol. 1, no. 2, 2012.

[11] M. F. Cabanas , F.P. Gonzales, M. G. Melero, C. H. Rojas, G. A. Orcajo, J. M. Cano and J. G. Norniella "Insulation fault diagnosisin high voltage power transformers by means of leakage flux analysis", Progr. Electromagn. Res., vol. 114, pp.211 -234 2011.

[12] L. M.R. Oliveira and A. J. M. Cardoso "A permeance based transformer modeland its application to winding interturn arcing fault studies", IEEE Trans. Power Del., vol. 25, no. 3, pp.1589 -1598 2010 . 